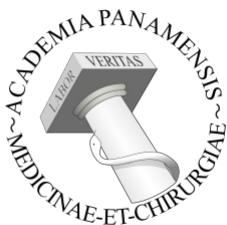

\title{
Informe de casos
}

\section{Explorando un seminoma, encontrado en una ruptura testicular: reporte de caso}

[Exploring a seminoma, found in a testicular rupture: a case report]

\section{Randol Gabriel Mojica Flores ${ }^{1}$}

${ }^{1}$ Hospital Joaquín Pablo Franco Sayas, Las Tablas, Panamá.

\section{Palabras Claves: \\ seminoma, testículo, orquiec- \\ tomía,trauma \\ Keywords: \\ seminoma, testicle, orchiectomy, \\ trauma \\ Correspondencia a: \\ Randol Gabriel Mojica Flores \\ Correo electrónico: \\ mojica0195@gmail.com \\ Recibido: \\ 01 de agosto de 2021 \\ Aceptado: \\ 10 de noviembre de 2021 \\ Publicado: \\ 24 de diciembre de 2021 \\ Aspectos bioéticos: \\ El autor declara que no existe conflicto de interés alguno asociado en la publicación de este manuscrito. La información cruda anonimizada se compartirá a solicitud por el autor co- rresponsal.}

Financiamiento:

El autor declara que no hubo

financiamiento para la realización de este trabajo.

Uso y reproducción:

Publicación de libre uso individual, no comercial. Prohibida la distribución para otros usos sin el consentimiento escrito del editorial.

\section{Resumen}

Dentro de los traumas testiculares, el cerrado representa la mayoría de los casos, y por lo general afecta a hombres de 15 a 40 años de edad. Presentamos un hallazgo de seminoma clásico luego de un trauma incidental con posterior exploración quirúrgica y orquiectomía radical de testículo izquierdo. Se resalta la dificultad de las herramientas de investigación como la ecografía testicular para apoyo del médico. Los seminomas suelen ser masas homogéneamente hipoecoicas. Las imágenes por resonancia magnética pueden ayudar a confirmar que una masa es intratesticular y proporcionar datos para la estadificación local. La tomografía computarizada proporciona información valiosa para la estadificación, incluida la presencia y el tamaño de los ganglios linfáticos retroperitoneales. El manejo es limitado. Sin embargo, el seminoma testicular se trata con orquiectomía inguinal radical y es altamente curable incluso en etapas avanzadas de la enfermedad. La mayoría de los médicos eligen la orquiectomía seguida de vigilancia para pacientes con enfermedad seminomatosa en estadio I y quimioterapia o radiación, seguida de una cirugía para el manejo de masas residuales, para pacientes con enfermedad en estadio II y superior. Destacamos la importancia de la sospecha clínica en estos tipos de pacientes jóvenes y tener una búsqueda activa ante estos traumas triviales.

\section{Abstract}

Among testicular traumas, blunt testicular trauma represents the majority of cases and usually affects men between 15 and 40 years of age. We present a finding of classic seminoma after an incidental trauma with subsequent surgical exploration and radical orchiectomy of the left testicle. The difficulty of research tools such as testicular ultrasound for physician support is highlighted. Seminomas are usually homogeneously hypoechoic masses. Magnetic resonance imaging can help confirm that a mass is intratesticular and provide data for local staging. Computed tomography provides valuable information for staging including the presence and size of retroperitoneal lymph nodes. Management is limited. However, testicular seminoma is treated with radical inguinal orchiectomy and is highly curable even in advanced stages of the disease. Most physicians choose orchiectomy followed by surveillance for patients with stage I seminomatous disease and chemotherapy or radiation, followed by surgery for management of residual masses, for patients with stage II and higher disease. We stress the importance of clinical suspicion in these types of young patients and having an active search for these trivial traumas.

INTRODUCCIÓN traumatismo escrotal representa menos del $1 \%$ de todas las lesiones relacionadas con el trauma, debido a la ubicación anatómica y la movilidad del escroto [1]. La rupEl traumatismo testicular se puede clasificar etiológica- tura testicular ocurre en el $50 \%$ de los casos de trauma mente en contundente, penetrante o deglomeración. El escrotal directo y contundente. La compresión intensa

Órgano oficial de la Academia Panameña de Medicina y Cirugía y del Instituto Commemorativo Gorgas para Estudios de la Salud (ICGES). Indexado en LILACS, EBSCO, y www.revistasmedicas.org.
R M P RMDP 2021; 41(3):60-64 
Mojica, R, Explorando un seminoma, encontrado en una ruptura testicular

de los testículos contra el ramo púbico inferior o la sínfisis resulta en una ruptura de la túnica albugínea. Se necesita una fuerza de aproximadamente $50 \mathrm{~kg}$ para causar ruptura testicular [2]. Los pacientes deben ser atendidos de forma rápida y llegar a un diagnóstico temprano para evitar en la medida de lo posible una pérdida testicular.

Presentamos un caso de seminoma clásico incidental tras ser diagnosticado por una exploración quirúrgica del testículo izquierdo como resultado de un trauma contuso trivial. La ruptura intraescrotal de cáncer testicular es rara y se han informado aproximadamente 6 casos en los últimos 65 años.

\section{Caso Clínico}

Se presenta un paciente masculino de 24 años de edad, con antecedente de trauma testicular cerrado 2 meses previo a la consulta, tras una patada mientras jugaba fútbol. Actualmente refiere historia de un segundo trauma ocasionado por un manotazo, mientras dormía 12 horas antes de la consulta. Posteriormente refiere dolor súbito e intenso 10/10 en región escrotal izquierda, que empeora con la bipedestación y al sentarse y mejora con el decúbito supino.

El dolor se irradia hacia ambas fosas iliacas, dejándolo con incapacidad para caminar. No refiere hematuria. Es llevado para atención primaria donde se trata con hidratación y analgésicos y luego lo envían a su casa. Doce horas después se reagudiza el cuadro clínico por lo que acude al servicio de urgencias de nuestra institución para reevaluación médica.

Paciente con antecedentes personales patológicos de asma desde los 8 años, no se acuerda de su última crisis y tratamiento previo. Consumo de alcohol desde los 19 años con aproximadamente 1 caja de cerveza los fines de semana, niega tabaco y uso de drogas. Se dedica a la agricultura. En cuanto a los antecedentes heredofamiliares niega alguna patología, niega cirugías, alergias, transfusiones, hospitalizaciones y medicamentos.

A la exploración física se observa aumento de volumen escrotal. Dolor a la palpación del hemiescroto izquierdo, con teste no palpable y transiluminación escrotal negativa para hematocele.

Piel de color eritematosa con leve tinte azulado en hemiescroto izquierdo. El teste derecho es de tamaño y consistencia normal. Signo de Prehn negativo.

Por la historia clínica del paciente y el examen físico se empieza a trabajar con un diagnóstico diferencial de trauma testicular cerrado a descartar ruptura testicular por lo que se inician los estudios de laboratorio y de gabinete pertinentes al caso.

El paciente cuenta con una ecografía doppler testicular (ver figura 1) posterior a su primer evento traumático donde se encuentran los siguientes hallazgos:

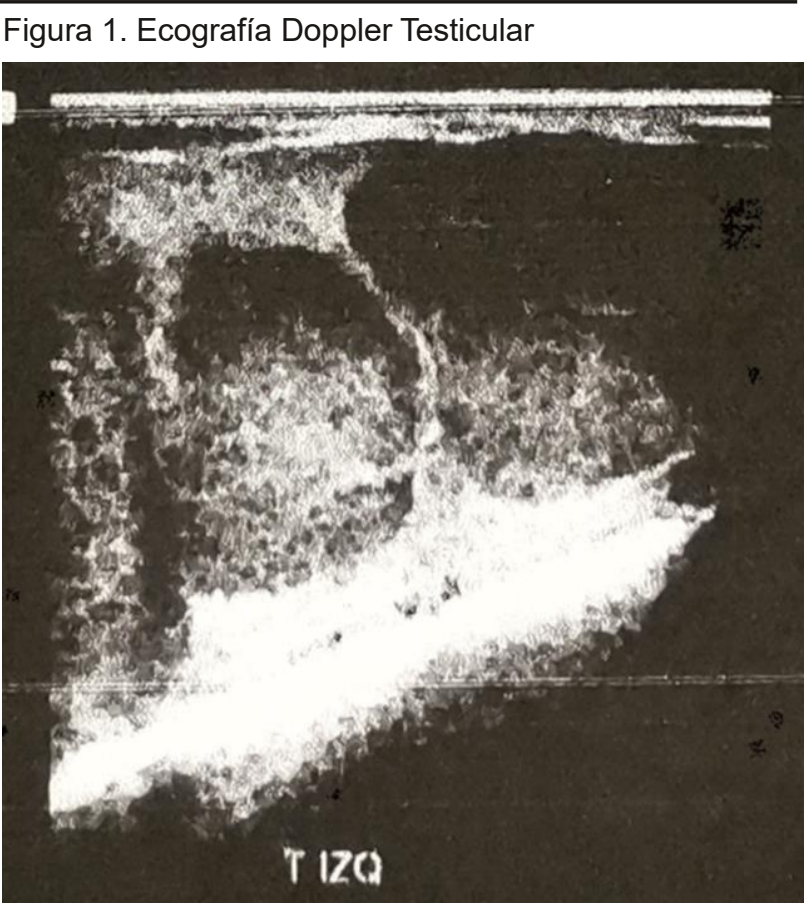

Lesiones Focales nodulares ubicadas en polo inferior de testículo izquierdo.

- Testículo izquierdo aumentado de volumen con marcada heterogeneidad y lesiones focales nodulares.

\section{- Testículo derecho sin alteraciones}

- Ausencia de hidrocele y varicocele

Consecuentemente por los hallazgos nodulares encontrados en su primera ecografía doppler testicular se le ordenan marcadores tumorales para descartar neoformaciones:

$$
\begin{aligned}
& \text { - Alfa fetoproteína: }<5.0 \mathrm{ng} / \mathrm{ml} \\
& \text { Valor de referencia: } 5-350 \mathrm{ng} / \mathrm{ml} \\
& \text { - BhCG Cuantitativa: } 7.83 \mathrm{mlU} / \mathrm{ml} \\
& \text { Negativo para: } 0-29 \mathrm{mlU} / \mathrm{ml}
\end{aligned}
$$

Para el curso del actual trauma se revisan las pruebas de laboratorio donde se observa leucocitosis con neutrofilia asociado al evento inflamatorio agudo del paciente por el trauma ocasionado, sin otros hallazgos relevantes. En el ultrasonido doppler testicular realizado en nuestro hospital revela un testículo izquierdo marcadamente aumentado de tamaño de aspecto heterogéneo, pero con vascularidad, asociado a aumento del epidídimo. (Ver figura 2)

Por estos hallazgos y la clínica del paciente se decide manejo médico iniciando antibioticoterapia con clindamicina $600 \mathrm{mg}$ intravenoso cada 8 horas y ciprofloxacina $200 \mathrm{mg}$ intravenoso cada 12 horas y tratamiento para el dolor con diclofenaco $50 \mathrm{mg}$ vía oral cada 8 horas. Se 
Figura 2. Vascularidad testicular pobre

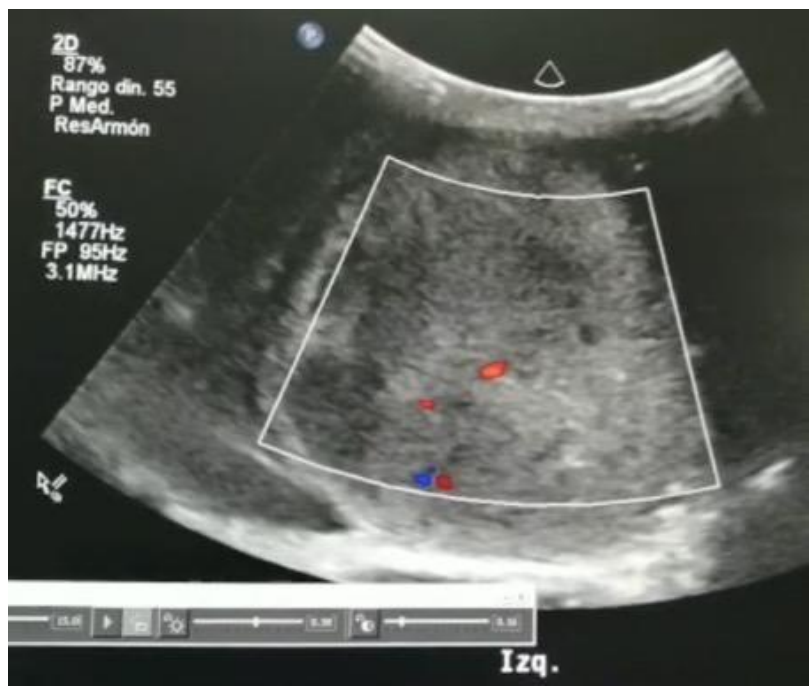

Presencia de vascularidad asociado a un aspecto heterogéneo en teste izquierdo.

Figura 3. Edema Tensional Escrotal

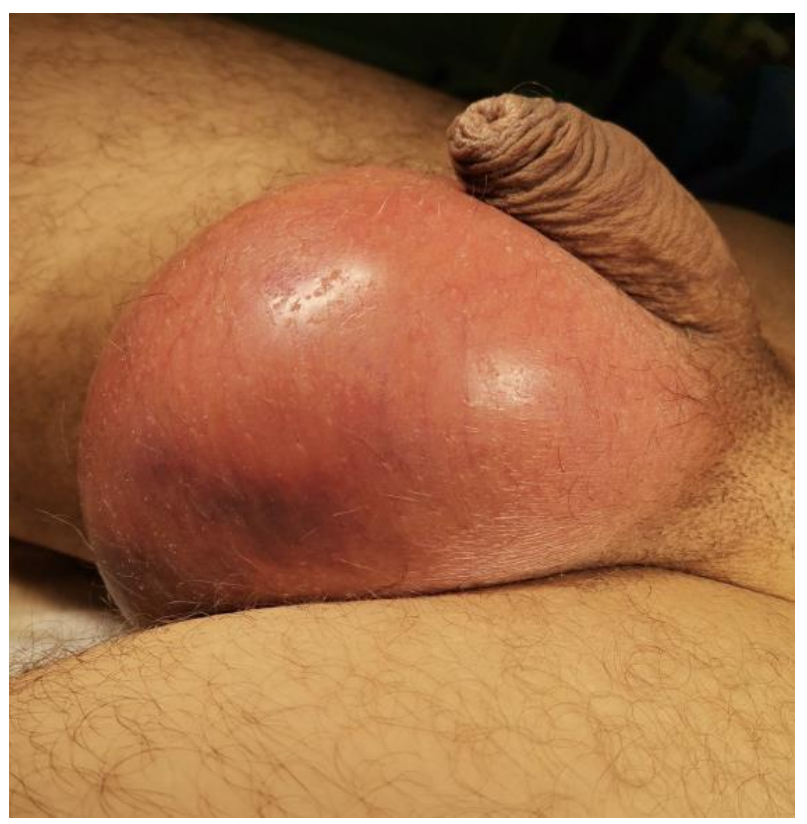

Aumento de toda la bolsa escrotal a las 48 horas de evolución, de color eritematoso con decoloración violácea en hemisferio escrotal izquierdo.

deja en observación por 48 horas, sin embargo, persiste con dolor, aumento de volumen escrotal a tensión (Ver figura 3) y cambio en la decoloración de la piel de tono cianógeno por lo que se decide una exploración quirúrgica para evaluar el estado del testículo izquierdo y considerar o no una probable orquiectomía parcial o total.

Durante la cirugía se encontró un hematoma circundante al testículo izquierdo, insalvable con ruptura sagital desde su tercio superior hacia inferior. Se palparon 2 masas de consistencia pétrea, con formas ovaladas bien circunscritas y vascularizadas. (Ver figura 4).

Se extirpa el tejido no viable y se realiza extracción de
Figura 4. Ruptura testicular

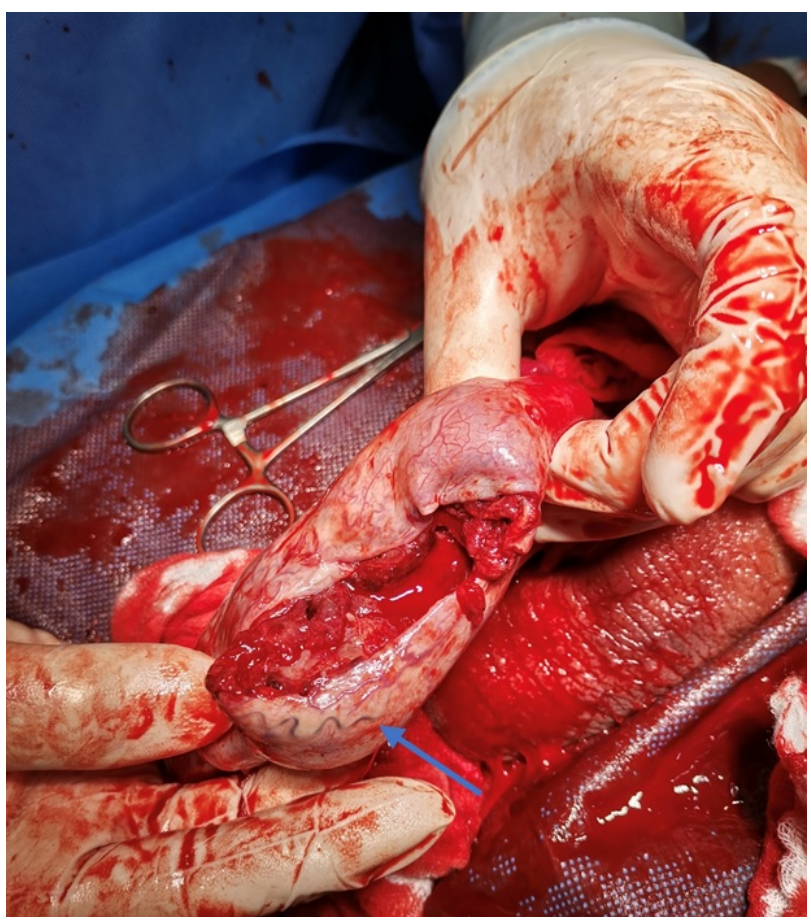

Se observa ruptura testicular asociado a vascularización tortuosa, con cambios en el calibre y color ubicado en el polo inferior de teste izquierdo.

todo el testículo izquierdo hasta el cordón espermático (orquiectomía total izquierda). Se sutura y se cierra por planos. Se manda la muestra hacia patología para los estudios pertinentes. La recuperación postoperatoria del paciente fue sin complicaciones.

\section{Discusión}

Este joven de 24 años ha tenido un trauma súbito en su bolsa escrotal y presentado un aumento progresivo de volumen y decoloración cianógena en las 48 horas de evolución, estos síntomas podrían ser consistentes con ruptura testicular.

\section{Ruptura Testicular}

La mayoría de las rupturas testiculares son diagnosticadas secundarias a lesiones relacionadas a deportes [3] con mayor frecuencia por golpe directo a la ingle. Aunque nuestro paciente fue golpeado en la actualidad por un manotazo trivial no relacionado a un deporte cabe resaltar que tuvo un antecedente traumático previo cuando jugaba fútbol.

Otras de las causas que pudieran provocar una ruptura testicular son accidentes vehiculares de motor como: carros y motocicletas además de caídas y golpes con dirección hacia la ingle. Sin embargo, el análisis de la literatura ha revelado pocos casos de rupturas testiculares relacionadas con tumores testiculares [4]. En estos casos se ha llegado al diagnóstico precedido por un trauma trivial y se plantea la cuestión sobre si la malignidad aumenta la probabilidad de ruptura testicular. Por la edad del paciente tanto la ruptura testicular como las 
Mojica, R, Explorando un seminoma, encontrado en una ruptura testicular

\begin{tabular}{|c|c|c|c|c|c|c|c|c|}
\hline Referencia & Año & Edad & $\begin{array}{l}\text { Diagnóstico } \\
\text { Inicial }\end{array}$ & $\begin{array}{l}\text { Historia de } \\
\text { trauma }\end{array}$ & Tratamiento & Histología & $\begin{array}{l}\text { Tratamiento } \\
\text { Adyuvante }\end{array}$ & Seguimiento \\
\hline Cassie (7) & 1956 & 22 & Hematocele & $\begin{array}{l}\text { Trauma } \\
\text { trivial } 2 \\
\text { semanas } \\
\text { antes }\end{array}$ & $\begin{array}{l}\text { Exploración } \\
\text { escrotal seguida de } \\
\text { orquiectomía } \\
\text { inguinal }\end{array}$ & Seminoma & Radioterapia & NR \\
\hline Cujatar (8) & 1972 & 18 & $\begin{array}{l}\text { Torsión } \\
\text { testicular }\end{array}$ & No & $\begin{array}{l}\text { Orquiectomía } \\
\text { inguinal }\end{array}$ & Teratoma & Radioterapia & 18 meses \\
\hline Liu $(9)$ & 2001 & Neonato & $\begin{array}{l}\text { Torsión } \\
\text { testicular }\end{array}$ & No & $\begin{array}{l}\text { Orquiectomía (no } \\
\text { especificada) }\end{array}$ & $\begin{array}{c}\text { Teratoma quístico } \\
\text { maduro }\end{array}$ & NR & 8 meses \\
\hline $\begin{array}{l}\text { La Montagne } \\
\text { (10) }\end{array}$ & 2002 & 19 & $\begin{array}{l}\text { Tumor } \\
\text { testicular }\end{array}$ & No & $\begin{array}{c}\text { Orquiectomía } \\
\text { inguinal }\end{array}$ & $\begin{array}{c}\text { Tumor } \\
\text { predominantemente } \\
\text { del saco vitelino }\end{array}$ & Quimioterapia & 6 meses \\
\hline Luchey (11) & 2009 & 24 & $\begin{array}{l}\text { Ruptura } \\
\text { testicular }\end{array}$ & $\begin{array}{c}\text { Aplastado } \\
\text { por árbol }\end{array}$ & $\begin{array}{l}\text { Orquiectomía } \\
\text { inguinal }\end{array}$ & $\begin{array}{l}\text { Tumor de células } \\
\text { germinales mixto }\end{array}$ & Quimioterapia & 12 meses \\
\hline Rahul $^{(4)}$ & 2014 & 42 & $\begin{array}{l}\text { Ruptura } \\
\text { testicular }\end{array}$ & $\begin{array}{c}\text { Trauma } \\
\text { trivial } 24 \\
\text { horas antes }\end{array}$ & $\begin{array}{l}\text { Orquiectomía } \\
\text { inguinal }\end{array}$ & Seminoma clásico & Quimioterapia & 12 meses \\
\hline
\end{tabular}

NR: no registrado.

malignidades testiculares se presentan en hombres jóvenes lo cual debe sospecharse activamente en aquellos que presentan este tipo de lesiones. En pacientes que se opte por el manejo conservador debe realizarse pruebas de marcadores tumorales: alfa fetoproteína (AFP), gonadotropina coriónica humana ( $\mathrm{hCG}$ ) para descartar neoformaciones como se realizaron en este paciente.

En el diagnóstico anatomopatológico de nuestro paciente se concluye:

- Seminoma clásico

- Roto con necrosis hemorrágica

- No se observa invasión vascular

- Se observan libres de tumor las siguientes estructuras: túnica albugínea, rete testis, epidídimo y cordón espermático.

\section{Seminoma}

El seminoma testicular es un diagnóstico patológico en el que solo se observan elementos seminomatosos tras la revisión histopatológica después de una orquiectomía radical y en el que el nivel sérico de alfafetoproteína (AFP) está dentro del rango de referencia [5]. El cáncer testicular, aunque raro, es la neoplasia maligna más común en hombres de 20 a 34 años; el $95 \%$ de los tumores malignos que surgen en los testículos son tumores de células germinativas (TCG), y los seminomas son el tipo más común de TCG testiculares [6].

La ruptura intraescrotal de un cáncer testicular es poco común (Ver tabla 1) y no existe un consenso sobre el manejo de esta patología en un escenario posterior a la orquiectomía, ya que el tratamiento primario es la orquiectomía inguinal radical. Además, se debe considerar un abordaje quirúrgico en los casos de ruptura testicular consecuentes a traumatismos leves cuando exista la sospecha de una malignidad asociada. Los hallazgos ecográficos del teste izquierdo mostraron una probable ruptura en la túnica albugínea y posiblemente un gran he-

matoma circundante con un patrón ecogénico mixto que dificultaba la observación del seminoma subyacente.

Para complementar el caso se le realiza una tomografía tóraco - abdómino - pélvica contrastada luego de 2 meses de la resección, para descartar metástasis pulmonares y lesiones ganglionares retroperitoneales tumorales en el paciente. Se reporta que no hay lesiones demostrables en la tomografía.Adjuntamente, se le realizaron marcadores tumorales para el correcto estadiaje, los cuales estuvieron dentro de los parámetros de referencia.

- BHCG Cuantitativa: $<0.100 \mathrm{mlU} / \mathrm{ml}$

- Alfa-fetoproteína: $2.58 \mathrm{UI} / \mathrm{ml}$

- LDH: $136 \mathrm{U} / \mathrm{L}$

Según el Comité Conjunto Americano sobre el Cáncer y la Unión Internacional contra el Cáncer [12], nuestro paciente se coloca en el siguiente estadiaje:

- Estadio I a (T1 N0 M0 S0)

\section{CONCLUSIONES}

Es un caso poco común donde nos demuestra la importancia de mantener siempre nuestra búsqueda activa y resaltar nuestras sospechas diagnósticas de una patología asociada para una lesión traumática contusa incidental en el escroto que puede terminar en presencia de neoplasias. Aunque tengamos nuestras herramientas diagnósticas, como lo es la ecografía, estas no pueden remplazar nuestro criterio clínico ya que tumores de pequeños tamaños pueden ser enmascarados por el daño del tejido parenquimatoso circundante al testículo. Por lo cual en algunos casos se debe proceder con la exploración quirúrgica para la correcta evaluación y estado de los testículos y verificar si puede o no haber un tumor relacionado. 


\section{REFERENCIAS}

[1] Rao, M. S., \& Arjun, K. (2012). Sonography of scrotal trauma. The Indian Journal of Radiology \& Imaging, 22(4), 293-297. DOI: https://doi.org/10.4103/09713026.111482

[2] EAU guidelines: Urological Trauma. (January 31, 2015). Uroweb.Org. https://uroweb.org/guideline/ urological-trauma/.

[3] Michael S Runyon, MD, MPH. (2021). Blunt genitourinary trauma: Initial evaluation and management. Apr 05, 2021, UpToDate, website: https://www.uptodate.com/contents/bluntgenitourinary-trauma-initial-evaluation-andmanagement.

[4] Lunawat, R., Craciun, M., Omorphos, S., Weston, P., \& Biyani, S. (2014). Seminoma presented as testicular rupture: Case report and literature review. Canadian Urological Association Journal, 8(9-10), 749. https://doi.org/10.5489/cuaj.2194. DOI: https:// doi.org/10.5489/cuaj.2194

[5] Testicular Seminoma: Practice Essentials, Background, Pathophysiology. Emedicine.medscape.com. (2021). Retrieved 30 July 2021, from https://emedicine.medscape.com/article/ 437966-overview. Accessed: July 6, 2021.

[6] Gilligan, T., Lin, D. W., Aggarwal, R., Chism, D., Cost, N., Derweesh, I. H., Emamekhoo, H., Feldman, D. R., Geynisman, D. M., Hancock, S. L., LaGrange, C., Levine, E. G., Longo, T., Lowrance, W., McGregor, B., Monk, P., Picus, J., Pierorazio, P.,
Rais-Bahrami, S., Pluchino, L. A. (2019). Testicular Cancer, version 2.2020, NCCN clinical practice Guidelines in oncology. Journal of the National Comprehensive Cancer Network: JNCCN, 17(12), 1529-1554. DOI: https://doi.org/10.6004/ jnccn.2019.0058

[7] Cassie, G. F. (1956). Rupture of the testis: seminoma. British Journal of Urology, 28(3), 283. DOI: https:// doi.org/10.1111/j.1464-410X.1956.tb04772.x

[8] Cutajar, C. L. (1972). Spontaneous rupture of testicular teratoma. British Medical Journal, 1(5793), 154-155. DOI: https://doi.org/10.1136/bmj.1.5793.154

[9] Liu, S. P., Huang, S. W., Lin, K. H., Lin, M. C., \& Hsieh, J. T. (2001). Ruptured cystic teratoma of the testis in a neonate. Taiwan Yi Zhi [Journal of the Formosan Medical Association], 100(11), 779-781.

[10] LaMONTAGNE, A. E., Jr. (2002). Spontaneous rupture of A testicular tumor. The Journal of Urology, 1787-1788. DOI: https://doi.org/10.1097/00005392200204000-00051

[11] Luchey, A., Rogers, A., Saunders, S. E., Williams, H. J., Fooks, H. J., \& Zaslau, S. (2009). Blunt testicular trauma results in rupture of mixed germ cell tumor. The Canadian Journal of Urology, 16(6), 4955-4957.

[12] Amin, M. B., Edge, S. B., Greene, F. L., Byrd, D. R., Brookland, R. K., Washington, M. K., Gershenwald, J. E., Compton, C. C., Hess, K. R., \& Sullivan, D. C. (Eds.). (2018). AJCC Cancer Staging Manual. Springer International Publishing. 